\title{
Stability analysis for first-order nonlinear differential equations with three-point boundary conditions
}

\author{
Kamala E. Ismayilova \\ Communicated by Allaberen Ashyralyev
}

\begin{abstract}
In the present paper, we study a system of nonlinear differential equations with three-point boundary conditions. The given original problem is reduced to the equivalent integral equations using Green function. Several theorems are proved concerning the existence and uniqueness of solutions to the boundary value problems for the first order nonlinear system of ordinary differential equations with three-point boundary conditions. The uniqueness theorem is proved by Banach fixed point principle, and the existence theorem is based on Schafer's theorem. Then, we describe different types of Ulam stability: Ulam-Hyers stability, generalized Ulam-Hyers stability. We discuss the stability results providing suitable example.
\end{abstract}

Keywords. Three-point boundary conditions, Ulam-Hyers stability, existence and uniqueness, fixed point theorems.

2010 Mathematics Subject Classification. 34B37, 37C25, 37C75.

\section{Introduction}

Lately, stability theory for functional, differential, integral and integro-differential equations has been intensively studied. The most frequent types of citations are shown in the following examples $[1,2,4]$. Stability theory was first introduced from a well-known question raised by S. M. Ulam at the Mathematics Club of the University of Wisconsin in 1940: "When a solution of an equation differing slightly from a given one must be somehow near to the solution of the given equation?" (see e.g., [7]). The first partial answer to Ulam's question in the case of Cauchy's equation in Banach spaces was given by D.H.Hyers in 1941, and which is obtained property is nowadays called the Ulam-Hyers stability (see for more detail [8]). Afterwards, various generalizations of Ulam-Hyers stability are obtained. We show this with the following examples: Ulam-Hyers-Rassias stability, generalized Ulam-Hyers stability, generalized Ulam-Hyers-Rassias stability [3, 5, 6, 9, 11-16]. 
In the past few years, the study of differential equations with nonlocal boundary conditions has been an important field of mathematics, that has recently received much attention of researchers; the reader is referred to [10, 17-31]. Stability problems for non-local boundary conditions have been newly studied by several authors (see e.g., [32-35] and the references therein).

Here for the first time we investigate Ulam-Hyers stability for three-point boundary value problems. The rest of this paper is organized as follows. In Section 2, notations, problem statement, general definitions, remark and auxiliary lemmas are given, which will be used in the proof of main results. In Section 3, we discuss existence results for investigated boundary value problems. In Section 4, Ulam stability analysis results are obtained. In Section 5, an example illustrates the application for the Ulam-Hyers stability .

\section{Problem statement and preliminaries}

In this section, we give problem statement, general definitions, remark and lemmas which are used throughout this paper. We denote by $C\left([0, T], R^{n}\right)$ the Banach space of all continuous functions from $[0, T]$ into $R^{n}$ with the norm

$$
\|x\|=\max \{|x(t)|: t \in[0, T]\},
$$

where $|\cdot|$ is the norm in space $R^{n}$.

We concerned the existence, uniqueness and stability of the system of nonlinear differential equations of the type

$$
\dot{x}(t)=f(t, x(t)), t \in[0, T],
$$

subject to three point boundary conditions

$$
A x(0)+B x\left(t_{1}\right)+C x(T)=d,
$$

where $A, B, C$ are constant square matrices of order $n$ such that $\operatorname{det} N \neq 0$, $N=A+B+C, f:[0, T] \times R^{n} \rightarrow R^{n}$ is a given function, $d \in R^{n}$ is a given vector and $t_{1}$ satisfies the condition $0<t_{1}<T$.

Definition 2.1. For every $\varepsilon>0$, the function satisfies $y \in C^{1}\left([0, T], R^{n}\right)$

$$
|\dot{y}(t)-f(t, y(t))| \leq \varepsilon, t \in[0, T],
$$

where the function $f$ is defined in (1). Let $x \in C\left([0, T], R^{n}\right)$ be a solution of the problem (1)-(2). If there exists a nonzero positive constant $k$ such that

$$
|y(t)-x(t)| \leq k \varepsilon, t \in[0, T],
$$

then the problem (1)-(2) is said to be Ulam-Hyers stable. 
Definition 2.2. Let $y \in C^{1}\left([0, T], R^{n}\right)$ satisfies the inequality in (3) and $x \in C\left([0, T], R^{n}\right)$ is a solution of (1)-(2). If there is a function $\varphi_{f} \in C\left(R^{+}, R^{+}\right)$ with $\varphi_{f}(0)=0$ satisfying

$$
|y(t)-x(t)| \leq \varphi_{f}(\varepsilon), t \in[0, T],
$$

then the problem (1)-(2) is said to be generalized Ulam-Hyers stable.

Remark 2.3. A function $y \in C^{1}\left([0, T], R^{n}\right)$ is said to be a solution to (3) if and only if we can find a function $\varphi \in C\left([0, T], R^{n}\right)$ (dependent on $y$ ) such that

(i) $|\varphi(t)| \leq \varepsilon$ for all $t \in[0, T]$,

(ii) $\dot{y}(t)=f(t, y(t))+\varphi(t), t \in[0, T]$.

For simplicity, we can look at the following problem:

Lemma 2.4. Suppose $\mu \in C\left([0, T], R^{n}\right)$ and $\operatorname{det} N \neq 0$. Then the unique solution of the following problem

$$
\dot{x}(t)=\mu(t), \quad t \in[0, T],
$$

with three-point boundary conditions

$$
A x(0)+B x\left(t_{1}\right)+C x(T)=d,
$$

is given by

$$
x(t)=D+\int_{0}^{T} G(t, \tau) \mu(\tau) d \tau,
$$

where

$$
D=N^{-1} d, G(t, \tau)= \begin{cases}G_{1}(t, \tau), & t \in\left[0, t_{1}\right], \\ G_{2}(t, \tau), & t \in\left(t_{1}, T\right]\end{cases}
$$

such that

$$
G_{1}(t, \tau)=\left\{\begin{array}{lc}
N^{-1} A, & 0 \leq \tau \leq t, \\
-N^{-1}(B+C), & t<\tau \leq t_{1}, \\
-N^{-1} C, & t_{1}<\tau \leq T
\end{array}\right.
$$

and

$$
G_{2}(t, \tau)=\left\{\begin{array}{lc}
N^{-1} A, & 0 \leq \tau \leq t_{1}, \\
N^{-1}(A+B), & t_{1}<\tau \leq t \\
-N^{-1} C, & t<\tau \leq T
\end{array}\right.
$$


Proof. If function $x=x(\cdot)$ is a solution of the differential equation (1), then for $t \in(0, T)$

$$
x(t)=x_{0}+\int_{0}^{t} \mu(\tau) d \tau
$$

where $x_{0}$ is an arbitrary constant vector. Now we define $x_{0}$ so that, the function in equality (7) satisfies condition (5)

$$
x_{0}=N^{-1} d-N^{-1} B \int_{0}^{t_{1}} \mu(t) d t-N^{-1} C \int_{0}^{T} \mu(t) d t .
$$

Now in (7) we take into account the value $x_{0}$ determined from the equality (8) and yield

$$
x(t)=N^{-1} d-N^{-1} B \int_{0}^{t_{1}} \mu(t) d t-N^{-1} C \int_{0}^{T} \mu(t) d t+\int_{0}^{t} \mu(\tau) d \tau .
$$

Suppose that, $t \in\left[0, t_{1}\right]$. Then we can write the equality (9) as follows:

$$
\begin{aligned}
& x(t)=N^{-1} d-N^{-1} B\left(\int_{0}^{t} \mu(\tau) d \tau+\int_{t}^{t_{1}} \mu(\tau) d \tau\right) \\
& -N^{-1} C\left(\int_{0}^{t} \mu(\tau) d \tau+\int_{t}^{t_{1}} \mu(\tau) d \tau\right)-N^{-1} C \int_{t_{1}}^{T} \mu(t) d t+\int_{0}^{t} \mu(\tau) d \tau .
\end{aligned}
$$

We group similar terms and then simplify:

$$
\begin{aligned}
x(t)= & N^{-1} d+\left(E-N^{-1} B-N^{-1} C\right) \int_{0}^{t} \mu(\tau) d \tau \\
& -\left(N^{-1} B+N^{-1} C\right) \int_{t}^{t_{1}} \mu(\tau) d \tau \\
-N^{-1} C \int_{t_{1}}^{T} \mu(t) d t= & N^{-1} d+N^{-1} A \int_{0}^{t} \mu(\tau) d \tau \\
& -N^{-1}(B+C) \int_{t}^{t_{1}} \mu(\tau) d \tau-N^{-1} C \int_{t_{1}}^{T} y(t) d t,(10)
\end{aligned}
$$


where $E$ is an identity matrix. Let us define new function as follows:

$$
G_{1}(t, \tau)= \begin{cases}N^{-1} A, & 0 \leq \tau \leq t, \\ -N^{-1}(B+C), & t<\tau \leq t_{1}, \\ -N^{-1} C, & t_{1}<\tau \leq T .\end{cases}
$$

Equality (10) can be rewritten as integral equation (11), this implies that,

$$
x(t)=N^{-1} d+\int_{0}^{T} G_{1}(t, \tau) \mu(\tau) d \tau .
$$

Now assume that, $t \in\left(t_{1}, T\right]$. Then we can write the equality (9) as follows:

$$
\begin{gathered}
x(t)=N^{-1} d-N^{-1} B \int_{0}^{t_{1}} \mu(t) d t-N^{-1} C \int_{0}^{t_{1}} \mu(t) d t \\
-N^{-1} C\left(\int_{t_{1}}^{t} \mu(\tau) d \tau+\int_{t}^{T} \mu(\tau) d \tau\right)+\int_{0}^{t_{1}} \mu(t) d t+\int_{t_{1}}^{t} \mu(\tau) d \tau \\
=N^{-1} d+\left(E-N^{-1} B-N^{-1} C\right) \int_{0}^{t_{1}} \mu(t) d t+\left(E-N^{-1} C\right) \int_{t_{1}}^{t} \mu(\tau) d \tau \\
-N^{-1} C \int_{t}^{T} \mu(\tau) d \tau=N^{-1} d+N^{-1} A \int_{0}^{t_{1}} \mu(t) d t \\
N^{-1}(A+B) \int_{t_{1}}^{t} \mu(\tau) d \tau-N^{-1} C \int_{t}^{T} \mu(\tau) d \tau .
\end{gathered}
$$

We introduce a new function as follows:

$$
G_{2}(t, \tau)= \begin{cases}N^{-1} A, & 0 \leq \tau \leq t_{1}, \\ N^{-1}(A+B), & t_{1}<\tau \leq t, \\ -N^{-1} C, & t<\tau \leq T .\end{cases}
$$

Hence, if $t \in\left(t_{1}, T\right]$, then we can write the equality (9) as follows:

$$
x(t)=N^{-1} d+\int_{0}^{T} G_{2}(t, \tau) \mu(\tau) d \tau .
$$


Thus, the solution of the boundary value problem (4)-(5) can be shown as follows:

$$
x(t)=D+\int_{0}^{T} G(t, \tau) \mu(\tau) d \tau .
$$

We showed the validity of (6). Proof is completed.

Lemma 2.5. Assume that $f \in C\left([0, T], R^{n}\right)$. Then the function $x(t)$ is a solution of the boundary value problem (1)-(2) if and only if $x(t)$ is a solution of the integral equation

$$
x(t)=D+\int_{0}^{T} G(t, \tau) f(\tau, x(\tau)) d \tau .
$$

Proof. Let $x(t)$ be a solution of the boundary value problem (1)-(2). This lemma can be derived by a similar argument to Lemma 2.4. By checking directly we make sure that the solution of integral equation (12) satisfies the boundary value problem (1)-(2). Lemma 2.5 is proved.

\section{Existence results}

Let $P$ be an operator such that, $P: C\left([0, T], R^{n}\right) \rightarrow C\left([0, T], R^{n}\right)$ as

$$
P x(t)=D+\int_{0}^{T} G(t, \tau) f(\tau, x(\tau)) d \tau .
$$

Obviously, the problem (1)-(2) is equivalent to the fixed point problem $x=P x$. So, the problem (1)-(2) has a solution if and only if the operator $P$ has a fixed point. In Lemma 2.4, we use the most basic fixed point theorem named the contraction mapping principle and it uses the assumption:

(H1) There exists a continuous function $M(t) \geq 0$ such that

$$
|f(t, x)-f(t, y)| \leq M(t)|x-y|
$$

for each $t \in[0, T]$ and all $x, y \in R^{n}$.

Theorem 3.1. Assume that, the assumption (H1) holds, and

$$
L=T S M<1
$$


then the boundary-value problem (1)-(2) has a unique solution on $[0, T]$, where

$$
\begin{gathered}
M=\max _{[0, T]} M(t), \\
S=\max _{[0, T] \times[0, T]}\|G(t, \tau)\| .
\end{gathered}
$$

Proof. Setting $\max _{[0, T]}|f(t, 0)|=M_{f}$ and choosing $r \geq \frac{\|D\|+M_{f} T S}{1-L}$ we show that $P B_{r} \subset B_{r}$ where

$$
B_{r}=\left\{x \in C\left([0, T], R^{n}\right):\|x\| \leq r\right\} .
$$

For $x \in B_{r}$, we have

$$
\begin{gathered}
\|P x(t)\| \leq\|D\|+\int_{0}^{T}|G(t, \tau)|(|f(\tau, x(\tau))-f(\tau, 0)|+|f(\tau, 0)|) d \tau \\
\leq\|D\|+S \int_{0}^{T}\left(M|x|+M_{f}\right) d t \leq\|D\| \\
+S M r T+M_{f} T S \leq \frac{\|D\|+M_{f} T S}{1-L} \leq r .
\end{gathered}
$$

Now for any $x, y \in B_{r}$ we have

$$
\begin{gathered}
|P x-P y| \leq \int_{0}^{T} \mid G(t, \tau)(f(\tau, x(\tau))-f(\tau, y(\tau)) \mid d \tau \\
\leq \int_{0}^{T}|G(t, \tau)||f(\tau, x(\tau))-f(\tau, y(\tau))| d \tau \\
\leq S \int_{0}^{T} M(t)|x(t)-y(t)| d t \leq S M T \max _{[0, T]}|x(t)-y(t)| \leq S M T\|x-y\| \\
\|P x-P y\| \leq L\|x-y\| .
\end{gathered}
$$

or

It is seen that, $P$ is contraction by condition (13). So, the boundary-value problem (1)-(2) has a unique solution. 
Theorem 3.2. (Schafer's fixed point theorem). Let $X$ be a Banach space. Assume that, $G: X \rightarrow X$ is a completely continuous operator and the set $\rho=\{x \in X \mid x=\beta G x, 0<\beta<1\}$ is bounded. Then $G$ has a fixed point in $X$.

Now we apply Schafer's fixed point theorem and it uses the following assumption:

(H2) The function $f:[0, T] \times R^{n} \rightarrow R^{n}$ is continuous.

Theorem 3.3. Assume that there exists $\rho \in C\left([0, T], R^{+}\right)$such that $|f(t, x(t))| \leq$ $\rho(t), \forall t \in[0, T], x \in C\left([0, T], R^{n}\right)$ with $\sup _{t \in[0, T]}|\rho(t)|=\|\rho\|$. Then the boundary value problem (1)-(2) has at least one solution on $[0, T]$.

Proof. Since $f$ is continuous, the operator $P$ is continuous. Let $\phi \in C\left([0, T], R^{n}\right)$ be bounded subset. Then $\forall x \in \phi$ together with the given assumption $|f(t, x(t))| \leq$ $\rho(t)$, we get

$$
|P(x)(t)| \leq \sup _{t \in[0, T]}\left\{D+\int_{0}^{T}|G(t, \tau)||f(\tau, x(\tau))| d \tau\right\} .
$$

Hence,

$$
|P(x)(t)| \leq\|D\|+S T \rho .
$$

Thus,

$$
\|P(x)(t)\| \leq\|D\|+S T \rho=l .
$$

This shows that, $P$ is bounded. Now for $0<\tau_{1}<\tau_{2}<T$, we have

$$
\left|P(x)\left(\tau_{2}\right)-P(x)\left(\tau_{1}\right)\right| \leq\|\rho\|\left(\tau_{2}-\tau_{1}\right)
$$

which tends to zero as $\tau_{2} \rightarrow \tau_{1}$. We conclude that the mapping $P: C\left([0, T], R^{n}\right) \rightarrow$ $C\left([0, T], R^{n}\right)$ is completely continuous by Arzela-Ascoli theorem. We show that a set $\Omega=\left\{x \in C\left([0, T], R^{n}\right): x=\lambda P(x)\right.$, for some $\left.0<\lambda<1\right\}$ is bounded. Assume that, $x=\lambda P(x)$ for some $0<\lambda<1$. Then for each $t \in[0, T]$, we can write

$$
x(t)=\lambda D+\lambda \int_{0}^{T} G(t, \tau) f(\tau, x(\tau)) d \tau .
$$

From here

$$
\|x\| \leq\|D\|+S\|\rho\| T \text {. }
$$

Therefore, the set $\Omega$ is bounded. Since all conditions of Theorem 3.2 are satisfied, $P$ has at least one fixed point. So, there exists at least one solution for the problem (1)-(2) on $[0, T]$. 


\section{Ulam stability analysis results}

Lemma 4.1. For every $\varepsilon>0$, the function $y \in C^{1}\left([0, T], R^{n}\right)$ satisfies the inequality

$$
|\dot{y}(t)-f(t, y(t))| \leq \varepsilon,
$$

then $y$ is a solution of the inequality

$$
|y(t)-P y(t)| \leq S T \varepsilon .
$$

Proof. From Remark 2.3 (ii) and Lemma 2.5, we have

$$
y(t)=D+\int_{0}^{T} G(t, \tau)(f(\tau, y(\tau))+\varphi(\tau)) d \tau .
$$

Then by Remark 2.1 (i), we obtain

$$
\begin{aligned}
& y(t)-P y(t)=\int_{0}^{T} G(t, \tau)(f(\tau, y(\tau))+\varphi(\tau)) d \tau \\
&-\int_{0}^{T} G(t, \tau) f(\tau, y(\tau)) d \tau, \\
&|y(t)-P y(t)| \leq S \int_{0}^{T}|\varphi(\tau)| d \tau \leq S T \varepsilon .
\end{aligned}
$$

Clearly, the inequality (14) is satisfied. This proves our statement.

Theorem 4.2. If the condition (H1) is satisfied and $\operatorname{det} N \neq 0$ holds, then the problem (1)-(2) is Ulam-Hyers stable.

Proof.

$$
\begin{gathered}
|y(t)-x(t)|=\left|y(t)-\int_{0}^{T} G(t, \tau) f(\tau, x(\tau)) d \tau\right| \\
=|y(t)-P y(t)+P y(t)-P x(t)| \leq|y(t)-P y(t)| \\
+\left|\int_{0}^{T} G(t, \tau)(f(\tau, y(\tau))-f(\tau, x(\tau))) d \tau\right|
\end{gathered}
$$




$$
\begin{gathered}
\leq S T \varepsilon+S M T\|y(t)-x(t)\|, \\
(1-S M T)\|y-x\| \leq S T \varepsilon, \\
\|y-x\| \leq \frac{S T \varepsilon}{1-S M T} .
\end{gathered}
$$

Obviously, the problem (1)-(2) is Ulam-Hyers stable. So, by setting $\varphi_{f}(\varepsilon)=\frac{S T \varepsilon}{1-S M T}, \varphi_{f}(0)=0$, problem (1)-(2) is generalized Ulam-Hyers stable. The proof is completed.

\section{Example}

Consider the following system of differential equation

$$
\left\{\begin{array}{l}
\dot{x}_{1}=\sin \alpha x_{2} \\
\dot{x}_{2}=\frac{\beta\left|x_{1}\right|}{1+t^{2}}
\end{array}\right.
$$

subject to

$$
x_{1}(0)+x_{2}(0)-x_{2}\left(\frac{1}{2}\right)=1,-x_{1}\left(\frac{1}{2}\right)+x_{1}(1)+x_{2}(1)=0 .
$$

Obviously,

$$
\begin{gathered}
A=\left(\begin{array}{ll}
1 & 1 \\
0 & 0
\end{array}\right), B=\left(\begin{array}{cc}
0 & -1 \\
-1 & 0
\end{array}\right), C=\left(\begin{array}{ll}
0 & 0 \\
1 & 1
\end{array}\right), \\
A+B+C=\left(\begin{array}{ll}
1 & 0 \\
0 & 1
\end{array}\right) .
\end{gathered}
$$

For $t \in\left[0, \frac{1}{2}\right]$, we obtain

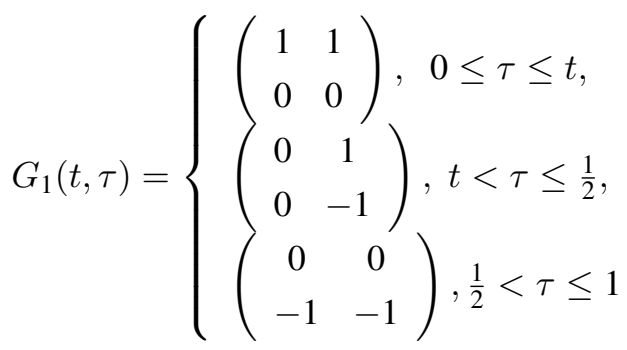


and for $t \in\left(\frac{1}{2}, 1\right]$

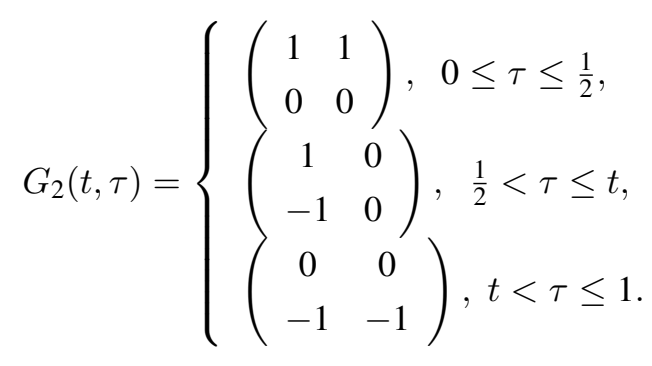

Here, $\|G\| \leq 2$ and $1 \cdot 2 \cdot \max \{|\alpha|,|\beta|\}<1$. So, $\max \{|\alpha|,|\beta|\}<\frac{1}{2}$. We can easily see that the given system is Ulam-Hyers stable.

\section{Bibliography}

[1] R. E. Bellman, Stability Theory of Differential Equations, McGraw-Hill, New York, Toronto, London, 1953.

[2] C. Bucur and E. Valdinoci, Nonlocal Diffusion and Applications, vol. 20 of Lecture Notes of the Unione Matematica Italiana, Springer, Basel, 2016.

[3] L. P. Castro and A. M. Simŏes, Hyers-Ulam and Hyers-Ulam-Rassias stability of a class of Hammerstein integral equations, Amer.Inst. Phys, AIP Conf. Proc. 1798(1) (2017), 020036, 10 pages.

[4] S. Şevgin and H. Şevli, Stability of a nonlinear Volterra integro-differential equation via a fixed point approach, J. Nonlinear Sci.Appl. 9 (2016), 200-207.

[5] S. M. Ulam, Problems in Modern Mathematics, John Wiley and Sons, New York, 1940.

[6] S. M. Ulam, A Collection of Mathematical Problems, Interscience, New York, 1968.

[7] S. M. Ulam, Problems in Modern Mathematics, Science Editions, John Wiley \& Sons, New York, 1960.

[8] D. H. Hyers, On the stability of the linear functional equation, Proc. Nat. Acad. Sci. U. S. A. 27(4) (1941), 222-224.

[9] L. P. Castro and A. M. Simŏes, Hyers-Ulam and Hyers-Ulam-Rassias stability of a class of integral equations on finite intervals, in CMMSE'17: Proceedings of the $17^{\text {th }}$ International Conference on Computational and Mathematical Methods in Science and Engineering, edited by J. Vigo-Aguiar (@CMMSE, Costa Ballena, Rota) I-VI (2017), 507-515.

[10] R. P. Agarwal, B. Ahmad, D. Garout and A. Alsaedi, Existence results for coupled nonlinear fractional differential equations equipped with nonlocal coupled flux and multi-point boundary conditions, Chaos Solit. Fract. 102 (2017), 149-161. 
[11] L. Cădariu, L. Găvruţa and P. Găvruţa, Fixed points and generalized Hyers-Ulam stability, Abstract and Applied Analysis 2012 (2012), 10 pages.

[12] R. Muralia and P. Selvan, Hyers-Ulam stability of $\mathrm{n}^{\text {th }}$ order linear differential equation, Proyecciones (Antofagasta, Online) 38(3) (2019), 553-566.

[13] T. M. Rassias, On the stability of linear mapping in Banach Spaces, Proc. Am. Math. Soc. 72 (1978), 297-300.

[14] T. M. Rassias, On a modified Hyers-Ulam sequence, J. Math. Anal. Appl. 158 (2003), $106-113$.

[15] M. Gachpazan and O. Baghani, Hyers-Ulam stability of nonlinear integral equation, Fixed Point Theory Appl. 2010 (2010), 927640, doi: 10.1155/2010/927640.

[16] I. A. Rus, Ulam stabilities of ordinary differential equations in a Banach space, Carpathian J. Math 26(1) (2010), 103-107.

[17] S. Asawasamrit, W. Nithiarayaphaks, S. K. Ntouyas and J. Tariboon, Existence and stability analysis for fractional differential equations with mixed nonlocal conditions, Mathematics 7(2) (2019), 11 pages.

[18] Y. A. Sharifov, Optimality conditions in problems of control over systems of impulsive differential equations with nonlocal boundary conditions, Ukrainian Mathematical Journal 64(6) (2012), 958-970.

[19] A. Ashyralyev and Y. A. Sharifov, Optimal control problem for impulsive systems with integral boundary conditions, AIP Conference Proceedings 1470(1) (2012), 1215 .

[20] A. Ashyralyev and Y. A. Sharifov, Optimal control problems for impulsive systems with integral boundary conditions, Electronic Journal of Differential Equations 2013(80) (2013), 11 pages.

[21] A. Ashyralyev and Y. A. Sharifov, Existence and uniqueness of solutions for nonlinear impulsive differential equations with two-point and integral boundary conditions, Advances in Difference Equations 2013(173) (2013), 11 pages.

[22] Y. A. Sharifov, Optimal control of impulsive systems with nonlocal boundary conditions, Russian Mathematics 57(2) (2013), 65-72.

[23] M. J. Mardanov, Y. A. Sharifov and H. H Molaei, Existence and uniqueness of solutions for first-order nonlinear differential equations with two-point and integral boundary conditions, Electron. J. Differ. Equations 2014(259) (2014), 1-8.

[24] M. J. Mardanov and Y. A. Sharifov, Existence results for first order nonlinear impulsive differential equations with nonlocal boundary conditions, AIP Conference Proceedings 1676(1) (2015), doi: 10.1063/1.4930441.

[25] M. J. Mardanov, Y. A. Sharifov and K. E. Ismayilova, Existence and uniqueness of solutions for nonlinear impulsive differential equations with three-point boundary conditions, e-Journal of Analysis and Applied Mathematics 2018(1) (2018), 21-36. 
[26] Y. A. Sharifov and K. E. Ismayilova, Existence and convergence of solutions to threepoint boundary value problems, Proceedings of the 6th International Conference on Control and Optimization with Industrial Applications vol. II, 11-13 July, (2018), Baku, Azerbaijan, 271-273.

[27] M. J. Mardanov, Y. A. Sharifov and K. E. Ismayilova, Existence and uniqueness of solutions for the first-order non-linear differential equations with three-point boundary conditions, Filomat 33(5) (2019), 1387-1395.

[28] M. J. Mardanov, Y. A. Sharifov, K. E. Ismayilova and S. A. Zamanova, Existence and uniqueness of solutions for the system of first-order nonlinear differential equations with three-point and integral boundary conditions, European Journal of Pure and Applied Mathematics 12(3) (2019), 756-770.

[29] M. J. Mardanov, Y. A. Sharifov and F. M. Zeynally, Existence and uniqueness of solutions for nonlinear impulsive differential equations with nonlocal boundary conditions, Vestn. Tomsk. Gos. Univ. Mat. Mekh. 60 (2019), 61-72.

[30] M. J. Mardanov, Y. A. Sharifov and F. M. Zeynalli, Existence and uniqueness of the solutions to impulsive nonlinear integro-differential equations with nonlocal boundary conditions, Proceedings of the Institute of Mathematics and Mechanics, National Academy of Sciences of Azerbaijan 45(2) (2019), 222-233.

[31] M. J. Mardanov, Y. A. Sharifov, R. A. Sardarova and H. N. Aliyev, Existence and uniqueness of solutions for nonlinear impulsive differential equations with threepoint and integral boundary conditions, Azerbaijan Journal of Mathematics 10(1) (2020), 110-126.

[32] J. Wang, M. Fečkan and Y. Tian, Stability analysis for a general class of noninstantaneous impulsive differential equations, Mediterr. J. Math. 14(46) (2017), 21 pages.

[33] J. Wang, A. Zada and W. Ali, Ulam's-type stability of first-order impulsive differential equations with variable delay in quasi-Banach spaces, International Journal of Nonlinear Sciences and Numerical Simulation 19(5) (2018), 553-560.

[34] A. Zada, O. Shah and R. Shah, Hyers Ulam stability of non-autonomous systems in terms of boundedness of Cauchy problems, Appl. Math. Comput. 271 (2015), 512-518.

[35] R. Shah and A. Zada, A fixed point approach to the stability of a nonlinear Volterra integrodifferential equation with delay, Hacet. J. Math. Stat. 47(3) (2018), 615-623.

Received December 27, 2019; revised April 8, 2020; accepted June 12, 2020.

\section{Author information}

Kamala E. Ismayilova, Baku Engineering University, Khirdalan city, 120, AZ0102, Absheron, Baku, Azerbaijan.

E-mail: keismayilova@beu.edu.az 